\title{
Optimization of the design of an integrated ultrasonic preamplifier
}

\author{
Johan Borg ${ }^{1}$, Jonny Johansson \\ EISLAB, Dept. of Computer Science and Electrical Engineering, \\ Luleå University of Technology, SE-971 87 Luleå, Sweden. \\ ${ }^{1}$ Email: johan.borg@1tu.se
}

\begin{abstract}
Traditionally BJT or BiCMOS amplifiers have been used to achieve equivalent input noise densities of $1 \mathrm{nV} / \sqrt{\mathrm{Hz}}$ or less, as desirable in some ultrasonic applications. Due to an increasing demand on increased integration it can be necessary to implement the amplifier in a CMOS process. As part of this design process we applied the particle swarm optimization to the problem of optimizing an amplifier specifically for operation in the $2-4 \mathrm{MHz}$ frequency band. We present measurements on the manufactured circuit with performance comparable to the best available BJT-based amplifiers available today.
\end{abstract}

Keywords: Low noise amplifier, Particle swarm optimization

\section{A Introduction}

The use of optimization techniques in IC design is nothing new, but the performance of the commonly used gradientbased optimization algorithms (e.g Levenberg-Marquardt or sequential quadratic programming) of a goal function does not work very well when the required derivatives are evaluated numerically from a "noisy" goal function. This is typically the case when the goal-function is based on the output from a circuit simulator, due to the limited numerical accuracy achieved when solving the large equation systems resulting when even quite small circuits are simulated. For small and well-behaved circuits gradient-based optimization methods can still be useful, but considerable fine-tuning of the parameters of the optimization algorithm may be required in order to ensure convergence.

This work presents the results of particle swarm optimization [1] applied to the problem of designing an areaconstrained and power constrained CMOS integrated low noise preamplifier. As the amplifier was intended to be used with $2-4 \mathrm{MHz}$ piezoelectric transducers, the gain, phase and noise optimization was performed only in that frequency band.

\section{B Particle swarm optimization}

In it's basic form an optimum is approximated by a set of "particles", each representing a point in the space where the goal function is defined. The procedure is carried out in iterations (or epochs), where the positions of all particles are updated according to their velocities. The velocities are calculated as the sum of the old velocity times an "inertia constant" and the distances to the positions of the best known solutions, both for the individual particle and for all particles, multiplied by scaling ("acceleration") constants and a random number with an uniform distribution between 0 and 1 . There exist several more or less specialized variations beyond the scope of this paper [2].

The choice of applying particle swarm optimization was based on the general nature of the algorithm. Any problem that can be described as a goal-function of any number of variables can be implemented with a limited number of parameters to be fine-tuned in order to get reasonable performance. Because each iteration of the algorithm calculates the goal function once for each particle before updating the particle positions, it is possible to calculate all goal functions in parallel, if desired. In our case this property was used for reducing the overhead when initializing the simulator, as all the simulations required for one iteration of the optimization could be grouped together.

For this paper we used the PSOt[3] Matlab ${ }^{\mathrm{TM}}$ implementation (common inertia) with a population of 50, an acceleration constant of 1.5 and an inertial weight 0.6 ramping down to 0.4 over 40 epocs.

\section{Amplifier design}

A design based on a two-stage shunt-series MOSFET based amplifier as shown in Fig.1 was selected as a test circuit. Since white noise dominates over the $1 /$ f noise in the relevant frequency range, an $\mathrm{N}$-mos input stage was chosen for the design, as the same transconductance, and thus noise, can be realized with a smaller transistor [4], with the additional benefit of lower input capacitance and thus better high-frequency performance.

In this test the voltage levels $V_{b 2}$ and $V_{b 3}$ are generated using current mirrors from external reference currents. $V_{b} 1$ and $V_{b 4}$ are generated using external voltage dividers. The DC-level of the input is set using an external low-frequency feedback loop forcing the output voltage to some preset level, typically $0.25 \mathrm{~V}$. The resistors $R_{s}, R_{f}$ and $R_{c}$ are implemented on-chip using poly-silicon resistors. The capacitor $C_{c}$ and additional decoupling capacitors (not shown in the schematic) for the supply voltage and the reference voltages were implemented using double-poly on-chip capacitors. The resistor $R_{l}$ is an external resistor (typically $100 \mathrm{Ohm}$, i.e. a $50 \mathrm{Ohm}$ resistor driving a $50 \mathrm{Ohm}$ coaxial cable). This resistor can alternatively be omitted for applications where this circuit is driv- 
ing a high-impedance input, with some reduction in power consumption.

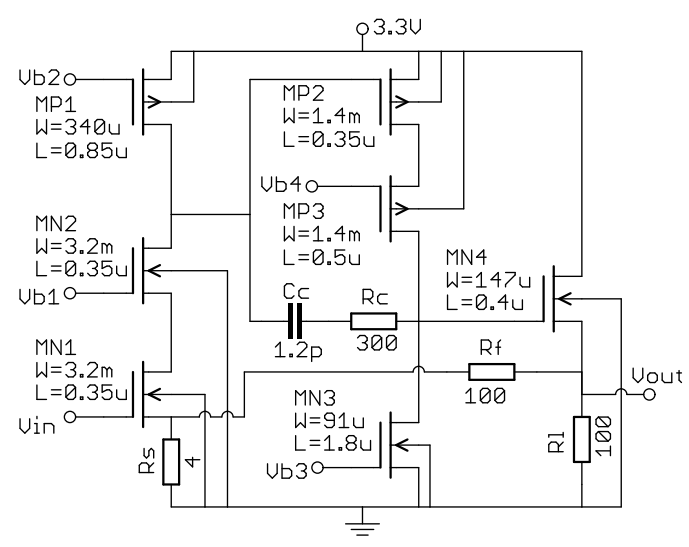

Fig. 1. Schematic of the circuit used

The following goal function $E$, to be minimized, was selected:

$$
\begin{array}{r}
E=\max \frac{d \phi_{o}}{d \omega} * W_{1}+\sqrt{\sum \phi_{o}\left(\omega_{k}\right)^{2}} * W_{2}+ \\
\left(\phi_{o}\left(\omega_{u}\right)-\phi_{m}\right) * W_{3}+N\left(\omega_{k}\right) * W_{4}+\frac{W_{5}}{A_{e}}+ \\
\frac{W_{6}}{\omega_{u}}+\left(A_{c}-A_{r}\right) * W_{7}+S * W_{8}
\end{array}
$$

where

- $W_{n}$ represent weights for each part

- $\phi_{o}$ the open loop phase (the derivative of which is included in order to enforce a monotonically decreasing open-loop phase)

- $\omega_{k}$ test frequencies ( 2 and $4 \mathrm{MHz}$ ) at which the phase and amplitude characteristics were evaluated

- $\omega_{u}$ represents the frequency at which the loop gain is one, assuming a closed loop gain of $A_{r}$

- $\phi_{m}$ is the desired phase margin

- $N\left(\omega_{k}\right)$ the equivalent input voltage noise

- $A_{e}$ the mean excess gain at the frequencies $\omega_{k}$

- $A_{c}$ the closed loop gain at the selected frequencies

- $A_{r}$ the requested closed loop gain.

- $S$ represents an approximation of the area required to implement the circuit.

It should be noted that the optimization generally did not reach the same "optimum" each time, which obviously means that the program failed to find a global optimum. We still found it useful for improving the circuit, even if each solution represented a different trade-off between the different parts of the goal-function.

A set of different trade-offs was generated by running the program several times with the weights $W=[1 \mathrm{e} 61 \mathrm{e}-$ $8 \mathrm{~s}^{-1} 1 \mathrm{e}-2 \mathrm{~s} 1 \mathrm{e} 9 \sqrt{\mathrm{Hz}} / \mathrm{V}$ 1e2 $1 \mathrm{e} 7 \mathrm{~Hz} 15 \mathrm{e} 6 \mathrm{~m}^{-2}$ ] and
$I_{t o t}=10 \mathrm{~mA}$, a voltage $V_{o}=0.25 \mathrm{~V}$ over the resistor $R_{o}$, a desired gain of 20 and a phase margin of 70 degrees with a source resistance of $100 \mathrm{Ohm}$. From this set the design with the component parameters shown in Fig.1 was chosen.

This specific design was implemented using an AMS analog 0.35um CMOS process and manufactured (Fig. 2) as part of an MPW through Europractice. The area required was $0.05 \mathrm{~mm}^{2}$ excluding bond pads.

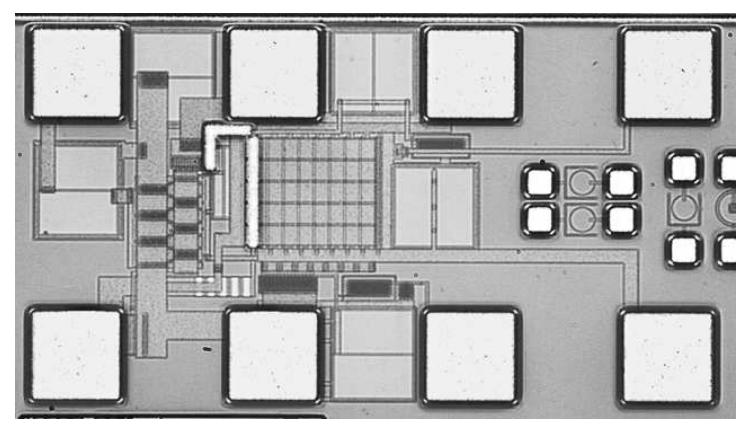

Fig. 2. The manufactured chip

\section{Results}

The manufactured chip was evaluated using a $R \& S^{\mathrm{TM}} \mathrm{ZVR}$ vector network analyzer for gain, impedance and delay measurements. A Tektronix ${ }^{\mathrm{TM}}$ TDS7254 oscilloscope was used for sampling the output signal resulting from presenting the input with different impedances. The equivalent input voltage and current noise sources are assumed to be uncorrelated. Only two chips have been evaluated so far.

With the exception of the noise performance data, which should be considered preliminary as no error analysis has yet been performed, enough averaging was applied to keep the measurement uncertainty below the guaranteed performance of the vector network analyzer, $0.2 \mathrm{~dB}$ amplitude and 1 degree phase accuracy.

As shown in Fig. 3 both the simulated and measured input noise levels are around $1 \mathrm{nV} / \sqrt{\mathrm{Hz}}$ at $4 \mathrm{MHz}$. The difference between simulated and measured performance is somewhat larger than expected, but can be attributed to sources unaccounted for in the simulations, such as gate, bulk and interconnect resistances.

Fig.4 shows the simulated equivalent input current noise. We have not been able to measure a current noise level significantly different from the estimated measurement uncertainty $(\approx 1 \mathrm{pA} / \sqrt{\mathrm{Hz}})$. This is not too surprising with the moderate input impedance and the very low expected noise level.

The difference between simulated and measured voltage gain (Fig.5) can probably be attributed to limited accuracy in the models used in the generation of small poly-silicon resistors, as used for the source and feedback resistances, in combination with ground bond-wire resistance, as the difference between the gain of two chips picked at random is small. The measured gain can also be claimed to be reasonably flat, although not as flat as in the simulations.

The input impedance (Fig.6) remains very high for all frequencies of interest. While the higher measured than 


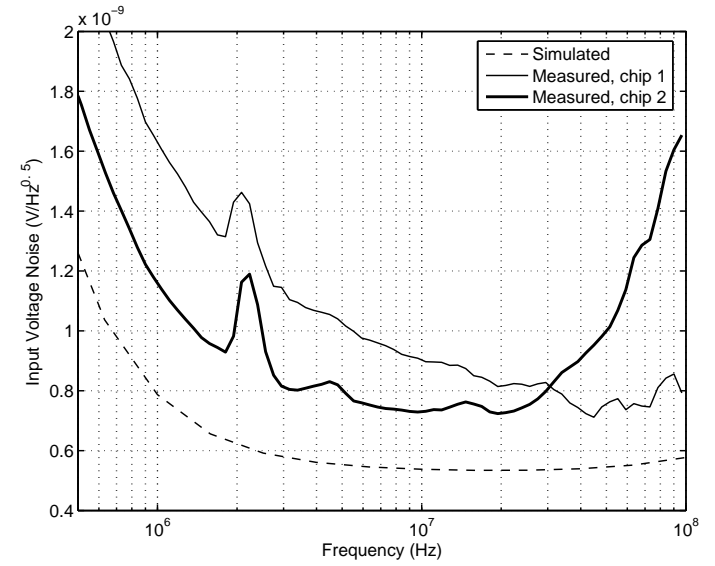

Fig. 3. Input Voltage Noise

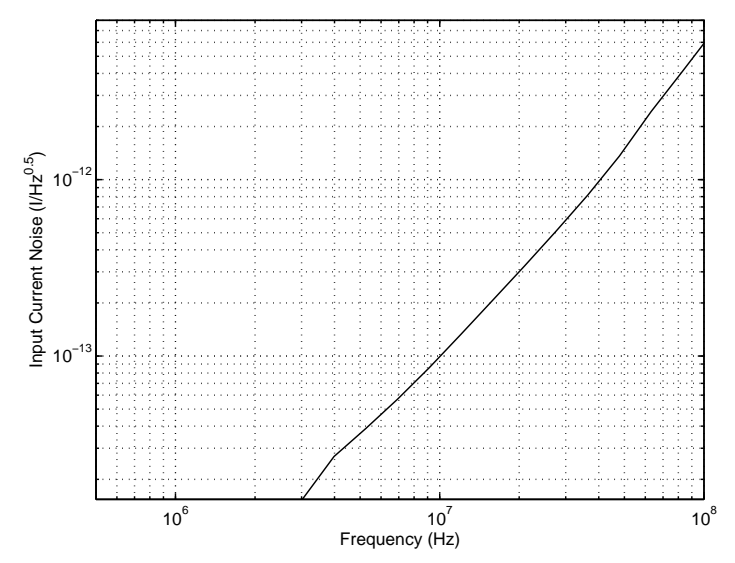

Fig. 4. Simulated Input Current Noise

simulated impedance is curious, it may partially be explained by the lower gain of the manufactured chips.

The amplifier does not remain stable for all possible input impedances at all frequencies, but is unconditionally stable for all frequencies of interest to us, as indicated by the input reflection coefficient (Fig.7, a input reflection coefficient larger than one corresponds to a negative input resistance). While the use of conditionally stable amplifiers is common practice in RF design, designing the amplifier to be unconditionally stable would have simplified it's use. The performance penalty of enforcing unconditional stability has not yet been determined. While the simulated and measured reflection coefficients differ considerably, it is interesting to note that the frequency from which instability is possible (i.e. where the reflection coefficient exceeds 1) and the peak reflection coefficient levels are very similar.

The output impedance (Fig.5) remains low enough for purposes such as driving a coaxial cable through a $50 \mathrm{Ohm}$ resistor with low return loss.

As shown in Table 1, from a low-power, low-noise amplifier perspective the present design compares well with commercial integrated amplifiers. It should however be remembered that those amplifiers posses several qualities not

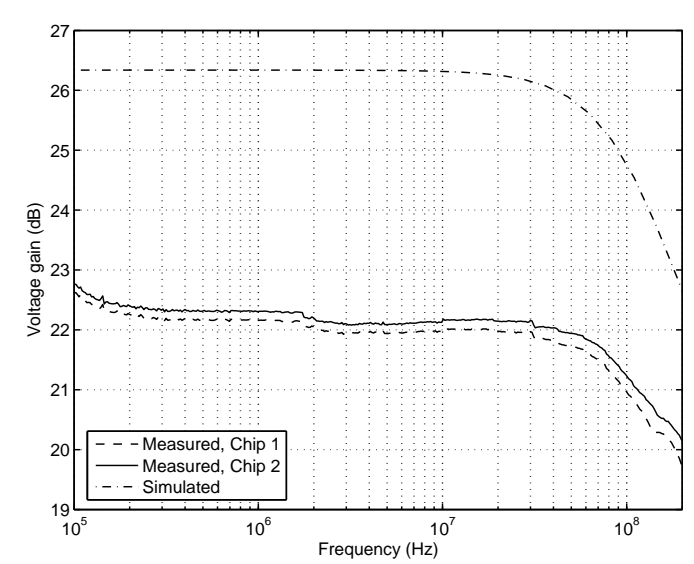

Fig. 5. Voltage Gain

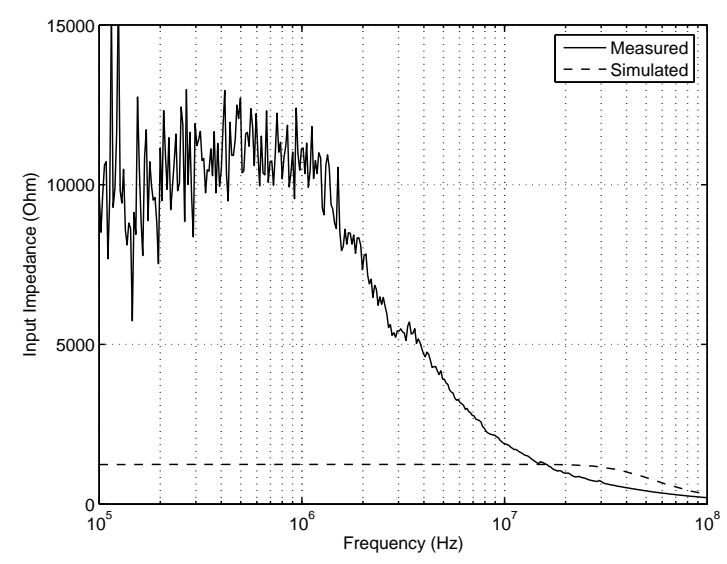

Fig. 6. Input Impedance

characterized (and expected to be inferior in this work), such as low-frequency noise, linearity, output level, bandwidth and the general versatility of operational amplifiers.

\section{E Conclusions}

We applied PSO to the problem of optimizing a low-noise amplifier for 2-4 MHz ultrasonic applications. While the convergence of the optimization is slow and tends to be towards local optimums, the stochastic nature of the optimization means that different, locally optimal solutions are found by running the optimizer several times. We thus found the method to be an improvement over the very tedious manual optimization otherwise required. It would be interesting to try to solve the same problem using other optimization methods.

The final design achieves similar noise performance as available commercial solutions, with a factor $30 \%$ reduction in power consumption compared to best known commercially available alternative (BJT-based), despite using a CMOS process, at the cost of higher specialization. 
Table 1. Low noise amplifier solutions

\begin{tabular}{|l|c|c|c|}
\hline $\begin{array}{l}\text { Part } \\
\#\end{array}$ & $\begin{array}{c}\text { Equivalent input voltage noise } \\
(\mathrm{nV} / \sqrt{\mathrm{Hz}})\end{array}$ & $\begin{array}{c}\text { Equivalent input current noise } \\
(\mathrm{p} A / \sqrt{\mathrm{Hz}})\end{array}$ & $\begin{array}{c}\text { Power consumption } \\
(\mathrm{mW})\end{array}$ \\
\hline MAX4106 & 0.75 & 2.5 & 150 \\
\hline LMH6624 & 0.92 & 2.3 & 60 \\
\hline AD797 & 0.9 & 2.0 & 246 \\
\hline LT1028 & 0.85 & 1.0 & 228 \\
\hline This work & 1.0 & (simulated to $\approx 40 \mathrm{fA})$ & 40 \\
\hline
\end{tabular}

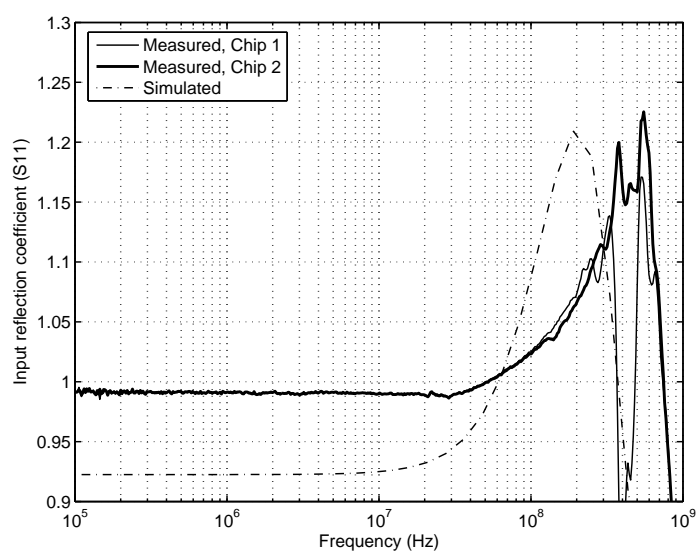

F Literature

[1] J. Kennedy and R. Eberhart, "Particle swarm optimization," 1995 IEEE International Conference on Neural Networks Proceedings (Cat. No.95CH35828), vol. vol.4, pp. 1942 8, 1995//.

[2] A. P. Engelbrecht, Fundamentals of computational swarm intelligence. John wiley \& sons inc, 2005.

[3] B. Birge, "Psot - a particle swarm optimization toolbox for use with matlab," Proceedings of the 2003 IEEE Swarm Intelligence Symposium. SIS'03 (Cat. No.03EX706), pp. 182 $6,2003 / /$

[4] C. D. Motchenbacker and J. Connely, Low-Noise Electronic System Design. John wiley \& sons inc, 1993.

Fig. 7. Input Reffection Coeffi cient

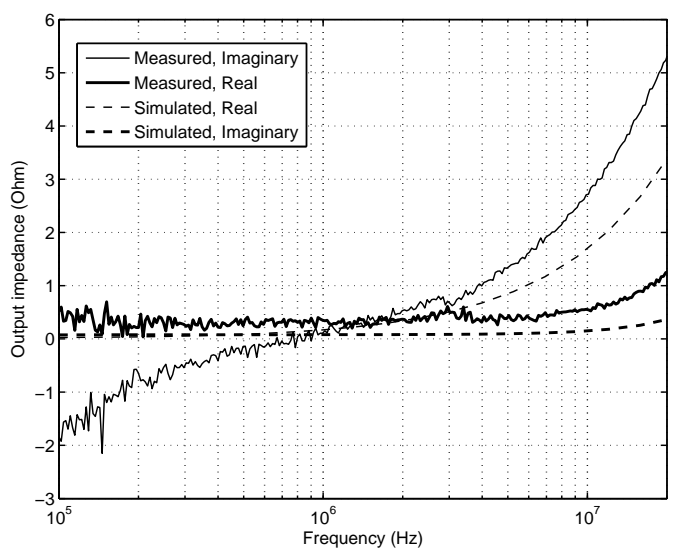

Fig. 8. Output Impedance 